\title{
ON SUBGROUPS OF FINITE SOLVABLE GROUPS
}

\author{
AVINOAM MANN ${ }^{1}$
}

In this note, the word "group" means "a finite solvable group." Let $G$ be a group, and $D$ a system normalizer of $G$. In [5] we introduced the subgroup $Q(D)$, generated by all subgroups of $G$ in which $D$ is subnormal. In this note we use one of the alternative characterizations of $Q(D)$, as given in [5], to define an analogue, $Q(H)$, for arbitrary subgroups $H$ of $G$. We derive a covering-avoidance characterization of $Q(H)$, and deduce that it is homomorphism invariant. These results, in turn, can be used to shorten many of the proofs in [5].

We first recall some definitions. A Sylow system $\subseteq$ of $G$ is said to reduce into $H$, if $\mathfrak{S} \cap H$ (i.e. the set of intersections of members of $\mathfrak{S}$ with $H$ ) is a Sylow system of $H$. An $H$-composition-series of $G$ is a series

$$
\{1\}=G_{n} \Delta G_{n-1} \triangle \cdots \triangle G_{1} \triangle G_{0}=G
$$

in which each $G_{i}$ is a maximal $H$-invariant normal subgroup of $G_{i-1}$. The groups $G_{i} / G_{i+1}$ are referred to as $H$-composition-factors of $G$. If $H$ induces (by conjugation) only the trivial automorphism on $G_{i} / G_{i+1}$, then the latter is $H$-central, otherwise it is $H$-eccentric. The product of the indices $\left|G_{i}: G_{i+1}\right|$, for those factors in (1) which are $H$-central and are avoided by $H$, is denoted by $z_{0}(H)$. Here a subgroup $K$ covers $G_{i} / G_{i+1}$ if $G_{i} \subseteq G_{i+1} K, K$ avoids $G_{i} / G_{i+1}$ if $G_{i} \cap K$ $\subseteq G_{i+1} \cdot z_{0}(H)$ is an invariant of $H$ (and $G$ ), i.e. it does not depend on the series (1) (see [2]).

Let $\mathfrak{M}$ be a set of Sylow systems of $G$. We refer to $\mathfrak{M}$ as a block, if $\mathfrak{M}$ is disjoint from all of its conjugates (so that if we consider $G$ as a permutation group on its Sylow systems, the conjugates of $\mathfrak{M}$ form an imprimitivity system).

Now let $H$ be any subgroup of $G$. We denote by $\mathfrak{M}_{0}$ the smallest block of $G$ which contains all the Sylow systems reducing into $H$.

Definition. The stabilizer of $\mathfrak{M}_{0}$ (i.e. the set of all $g \in G$ such that $\left.\mathfrak{M}_{0}=\mathfrak{M}_{0}\right)$ is denoted by $Q(H)$.

THeORem 1. $Q(H)$ covers all $H$-central $H$-composition-factors of $G$. Moreover, if $K \supseteq H$ and $K$ covers all $H$-central $H$-composition-factors, then $K \supseteq Q(H)$.

Received by the editors October 21, 1968.

1 This research was partially supported by National Science Foundation grant, GP-7952X. 
Proof. Let $G_{i} / G_{i+1}$ be an $H$-central factor in (1), and let $\subseteq$ be a Sylow system of $G$ reducing into $H$. Then $\mathfrak{S}$ reduces into $G_{i} H[\mathbf{3}$, Lemma 2.7]. Let $D$ be $N_{G_{i} I}\left(\Im \cap G_{i} H\right)$. Then $D$ transforms $S$ into systems reducing in to $H$ (because they all have the same intersection with $\left.G_{i} H\right)$, and thus $D$ stabilizes $m_{0}$, and $D \subseteq Q(H)$. Since $D$ covers the central factor $G_{i} / G_{i+1}$ of $G_{i} H, Q(H)$ covers $G_{i} / G_{i+1}$.

Now let $K \supseteq H$, and assume that $K$ covers all $H$-central factors. A $K$-central factor is certainly $H$-central, so $K$ covers all of its central factors, and thus $K$ is abnormal (see [2, §2]; an abnormal subgroup is one for which $g \in\left\langle K, K^{g}\right\rangle$ for all $\left.g \in G\right)$. The intersections of $K$ with the terms of (1) form an $H$-composition series of $K$, and as $K$ covers all $H$-central factors in (1), these give rise to $H$-central factors of $K$ of the same order. Thus $z_{0}(H)$, computed in $K$, is the same as $z_{0}(H)$, computed in $G$.

Let $D$ be a system normalizer of $G$, and $D_{1}$ one of $K$. By [2, p. 541] there are $|H| /|D| \cdot z_{0}(H)$ Sylow systems of $G$ reducing into $H$, $|H| /\left|D_{1}\right| \cdot z_{0}(H)$ systems of $K$ reducing into $H$, and each system of $K$ is the intersection with $K$ of $\left|D_{1}\right| /|D|$ systems of $G$. It follows that the number of systems of $G$ reducing into both $K$ and $H$ is

$$
\frac{\left|D_{1}\right|}{|D|} \cdot \frac{|H|}{\left|D_{1}\right|} \cdot z_{0}(H)=\frac{|H|}{|D|} \cdot z_{0}(H)
$$

i.e. all systems of $G$ reducing into $H$ reduce also into $K$. Let $\mathfrak{M}$ be the set of all Sylow systems reducible into $K$. Then, $K$ being abnormal, $\mathbb{M}$ is a block with stabilizer $K\left[\mathbf{5}\right.$, Lemma 2]. Thus $\mathbb{M}_{2} \supseteq \mathbb{M}_{0}$, and the stabilizer of $\mathfrak{M}_{0}$ is contained in the stabilizer of $\mathfrak{M}$.

REMARK 1. It is seen from the proof that it is enough to assume that $K$ covers the $H$-central factors in a given series (1).

REMARK 2. For each central factor $G_{i} / G_{i+1}$ in (1), let $D_{i}$ be a system normalizer of $G_{i} H$, as in the first paragraph of the proof. Then we have seen that $D_{i} \subseteq Q(H)$, and that $D_{i}$ covers $G_{i} / G_{i+1}$. Thus Theorem 1 implies that $Q(H)=\left\langle H, D_{i}\right\rangle$ (i ranges over all indices such that $G_{i} / G_{i+1}$ is $H$-central).

REMARK 3. Take $K=Q(H)$ in the above proof. Then $\mathfrak{M} \supseteq_{0} . \mathfrak{M}$ If $\Im \in \mathfrak{M}_{0}$ and $g \in Q(H)$, then $\mathfrak{S}^{g} \in \mathfrak{M}_{0}$. Take $\subseteq$ to reduce into $H$, then we have seen that $\mathfrak{S}$ reduces into $Q(H)$, and all systems reducing into $Q(H)$ are conjugate under $Q(H)$ by $\left[1\right.$, Lemma, p. 360]; thus $\mathfrak{M} \subseteq \mathfrak{M}_{0}$ and $\mathfrak{M}_{0}$ is the set of all Sylow systems reducing into $Q(H)$.

TheOREM 2. Let $G \rightarrow G^{*}$ be an epimorphism, and let stars denote epimorphic images. Then $Q\left(H^{*}\right)=Q(H)^{*}$.

Proof. Let $N$ be the kernel of the epimorphism, and let $R / N$ $=Q(H)^{*}, Q=Q(H)$. We may assume that $N$ is one of the terms in 
(1). Then $Q^{*}$ covers all $H^{*}$-central factors in the $H^{*}$-compositionseries $\left\{G_{i}^{*}\right\}$ of $G^{*}$. Thus $Q^{*} \supseteq R$. In turn, $R$ covers all $H$-central factors in (1), so $R \supseteq Q$, and $R^{*}=Q^{*}$.

Suppose $H \triangle \triangle L$, and let $\mathfrak{R}$ be the set of Sylow systems reducible into $L$. Then all systems in $\mathfrak{N}$ reduce into $H$, so $\mathfrak{R} \subseteq \mathfrak{M}_{0}$. As $L$ stabilizes $\mathfrak{N}, L \subseteq Q(H)$. In general, $Q(H)$ is not generated by all such $L$, as we can see by taking $H$ to be any self-normalizing subgroup that is not abnormal.

Now take $D$ to be any subgroup normalizing the Sylow system $\mathfrak{\subseteq}$ of $G$. In the notations of Remark 2, $D \subseteq D_{i}$ for each of the $i$ 's considered there. Thus $Q(D)=\left\langle D_{i}\right\rangle$, and $D \triangle \triangle D_{i}$, as each $D_{i}$ is nilpotent. So $Q(D)$ is generated by all subgroups in which $D$ is subnormal. If $D \subseteq E$ and $E$ is nilpotent, then $D \triangle \triangle E$, hence $E \subseteq Q(D)$. On the other hand, the subgroups $D_{i}$ are nilpotent. We thus see that $Q(D)$ is, indeed, the subgroup introduced in [5], and at the same time we have alternative proofs for the properties of $Q(D)$ discussed there (the present treatment is slightly more general, as we allow $D$ to be an arbitrary subgroup of a system normalizer).

As a further application, consider the problem: when is $\mathfrak{M}_{0}$ the set of all systems reducing into $H$ ? Suppose this is the case.By Remark 3, all systems of $Q(H)$ reduce into $H$, so that $H \triangle \triangle Q(H)$ [2] or [4]. We then have in $Q(H)$, and therefore also in $G, z_{0}(H)=|Q(H): H|$. Thus $Q(H)$ is the strong subnormalizer of $H$, in the sense of [5]. Conversely, assume that $H \triangle \triangle L$ and that $|L: H|=z_{0}(H) . L$ covers or avoids all factors in (1), and the ones that $L$ covers but $H$ avoids must be $H$-central (they are $H$-isomorphic to factors between $L$ and $H)$. By orders, $L$ covers all $H$-central factors, so $L \supseteq Q(H), L=Q(H)$, and $L$ is necessarily the strong subnormalizer of $H$. Since $H \triangle \triangle L$, all systems of $L$ reduce into $H$, so $\mathfrak{M}_{0}$ is indeed the desired set of Sylow systems. We have thus reproved Theorems 3 and 4 of [5], while Theorem 5 there follows from our present Theorem 2.

\section{REFERENCES}

1. J. L. Alperin, System normalizers and Carter subgroups, J. Algebra 1 (1964), 355-366.

2. R. W. Carter, Nilpotent self-normalizing subgroups and system normalizers, Proc. London Math. Soc. 12 (1962), 535-563.

3. - On a class of finite soluble groups, Proc. London Math. Soc. 9 (1959), 623-640.

4. O. H. Kegel, Sylow Gruppen und Subnormalteiler endlicher Gruppen, Math. Z. 78 (1962), 205-221.

5. A. Mann, System normalizers and subnormalizers, Proc. London Math. Soc. (to appear).

UNIVERSITY OF ILLINOIS, URBANA AND Institute for Advanced Study, Princeton 